\title{
Relationship between periodic limb movement and seizure recurrence in genetic generalized epilepsy
}

\author{
Mohamed Mohamed Hamdy, Amr Mohamed Elfatatry, Jaidaa Farouq Mekky and Eman Hamdy * (D)
}

\begin{abstract}
Background: Although periodic limb movements are prevalent among patients with epilepsy, the exact relationship between these movements and epilepsy remains elusive.

Objective: The aim of this work was to study the periodic limb movements among patients with genetic generalized epilepsy in relation to different clinical characteristics and seizure recurrence.
\end{abstract}

Methods: Sixty individuals participated in this study: 30 of them were newly diagnosed with genetic generalized epilepsy and 30 were healthy individuals. Sleep quality was assessed using the Pittsburgh Sleep Quality Index questionnaire, and a standard polysomnographic study was conducted to all subjects. All patients then received sodium valproate in therapeutic doses and were followed up for 6 months. Electroencephalogram (EEG) was done after the follow-up period and seizure recurrence was assessed. Accordingly, patients were classified into three groups according to clinical seizure recurrence and follow-up EEG findings. Periodic limb movement index and frequency were compared among the patients' groups and were correlated with different clinical characteristics.

Results: Periodic limb movement index was highest among the group who were not controlled, followed by the partially controlled, then the controlled group. The difference between the three groups was statistically significant.

Conclusion: Periodic limb movement index was positively correlated with seizure recurrence and can be used as a predictor of seizure outcome.

Trial registration: The trial registration number (TRN) on clinicaltrials.gov is NCT03587506, and the date of registration is July 16, 2018.

Keywords: Epilepsy, Sleep, Periodic limb movements, Seizure recurrence

\section{Introduction}

The relationship between sleep and epilepsy has been studied thoroughly over the last decades. Sleep influences the occurrence, frequency, and timing of interictal epileptiform discharge (IED) and clinical seizures. It also worsens the sleep architecture of epileptic patients. It is thus well-established that sleep and epilepsy are highly correlated with a vicious circle between them [1]. Sleep disorders are more common in patients with epilepsy than in the general population. For instance, up to one third of patients with medically refractory epilepsy have an apnea-hypopnea index of $>5$, and about $10 \%$ have a

\footnotetext{
* Correspondence: amygdala88@gmail.com

Department of Neurology and Psychiatry, Faculty of Medicine, Alexandria University, 12 Italy Hospital Street, Moharram Bik, Alexandria, Egypt
}

periodic limb movement index of $>20$ events per hour [2]. Insomnia occurs in about $11 \%$ of children and $35-45 \%$ of adults with epilepsy, and about $28-48 \%$ of patients with epilepsy report daytime sleepiness [3].

Periodic limb movements (PLMs) are repetitive periodicpseudoperiodic, stereotypical movements that consist of toe extension, ankle dorsiflexion, knee flexion, and sometimes hip flexion. Anterior tibialis surface electromyography (EMG) recoding of these movements shows bursts of muscle activity lasting for $0.5-10 \mathrm{~s}$. The contractions occur in episodes with each of them consisting of at least four contractions. The interval between the episodes lasts for 5-90 s. Periodic limb movement disorder is diagnosed when PLMs are associated with clinical history of sleep disturbance that is not attributed to other sleep disorders [4]. 
Although many central neurophysiological mechanisms have been suggested to explain the phenomena of PLMs, precise etiology remains elusive. The periodicity of these movements is thought to be regulated by endogenous cerebral and brain-stem-activated mechanisms [5]. It has been demonstrated that the PLMs are associated temporally with cortical (EEG) arousal and autonomic activation. However, the periodic EEG arousal and autonomic oscillations also exist in the absence of PLMs and are known as the cyclic alternating pattern. Thus, it is still unknown whether the temporal relationship between these events reflects a direct causal relationship or it is more complex and more in line with a reciprocal reinforce than a linear direct causality [6, 7]. Periodic limb movements are more prevalent in epileptic patients than the general population $[2,8]$. However, the relationship between periodic limb movement and epilepsy is not well-understood. The aim of this work was to study the relationship between periodic limb movement and seizure recurrence in epilepsy.

\section{Patients and methods Study design}

This was a prospective cohort study that was conducted in an outpatient clinic of the epilepsy unit at Alexandria University Hospital in Egypt. Patients were recruited during the period from March 2015 to September 2016

\section{Patients}

Sixty individuals participated in this study: 30 were patients and 30 were volunteer healthy individuals recruited as controls. All patients were newly diagnosed with genetic generalized epilepsy according to the ILAE 2010 criteria and the revised 2017 criteria [9] who did not previously receive AEDs. The clinical seizures were either eye-witnessed by physicians or video-recorded to ensure they were true epileptic seizures. Age of participants ranged from 9 to 29 years. All patients recruited in this study had at least two unprovoked seizures occurring $>24 \mathrm{~h}$ apart [10]. Patients with pre-existing psychiatric illnesses [11], those with seizures due to toxic or metabolic causes (including glucose disturbance, uremia, and electrolytes disturbance), infections, or structural causes (such as neoplasms), and those using hypnotic drugs were excluded. The control subjects were healthy volunteers who were not related to the patients and had no family history of epilepsy.

\section{Methods}

Data were collected from all subjects included age, gender, age at onset of epilepsy, family history of epilepsy, seizure type, frequency of seizure/s at onset of clinical presentation, diurnal variation of seizures, and ictal semiology. Sleep history was taken from all subjects using the Pittsburgh Sleep Quality Index questionnaire to assess the quality of sleep by measuring seven components: subjective sleep quality, sleep latency, sleep duration, habitual sleep efficiency, sleep disturbances, use of sleeping medications, and daytime dysfunction over the last month. Complete neurological examination was performed. Body mass index (BMI) was calculated for all individuals using the equation: $\mathrm{BMI}=$ body weight in $\mathrm{kg}$ )/height in meters $)^{2}$. Routine chemistry panel was performed for all subjects including complete blood picture (CBC), renal function tests (blood urea nitrogen (BUN), and creatinine level), liver enzymes (AST, ALT), electrolytes (sodium, potassium, calcium), and blood glucose level. Brain imaging was conducted to exclude structural etiology of epilepsy (CT scan or MRI in some cases). Baseline routine 16-channel electroencephalogram (EEG) was performed using a Nihon Kohden electroencephalograph (Neurofax EEG-1100, Japan) with at least 3-min hyperventilation and photic stimulation [12]. Polysomnography: all subjects underwent an overnight polysomnographic (PSG) studies by using the data acquisition system Cadwell (Version 2.1, USA). The studies were performed at the sleep laboratory of the department of neurology at Alexandria University Hospital. The standard PSG recordings were used which consisted of four channels of EEG, two lateral canthus eye leads for electrooculogram (EOG), two submental leads, two electrocardiogram (ECG) leads, one nasal thermistor for air flow, one channel for oxygen saturation, chest and abdominal belts to monitor respiratory movements, and two anterior tibialis electromyography leads to monitor leg movements.

The obtained sleep data were manually scored by two independent sleep specialists in 30-s epochs according to the American Academy Standard Manual (AASM) for the scoring of sleep-associated events (2014) [13]. The following sleep parameters were gathered for all subjects: time in bed (TIB), total sleep time (TST), sleep efficiency, wakefulness after sleep onset (WASO), sleep latency, REM latency, \% stages I and II, slow wave and REM, arousal index, total stage changes and stage changes per hour of sleep, snoring index, apnea-hypopnea index (AHI), and periodic limb movement index (PLM).

After performing all the previous investigations, all patients received sodium valproate [14] in therapeutic doses $(15-20 \mathrm{mg} / \mathrm{kg} /$ day on divided doses), and they were followed up for 6 months. During the follow-up period, patients recorded their seizures (if any occurred) in already prepared sheets. All seizure types were recorded either minor seizures such as absence seizures or myoclonic jerks or major seizures such as the generalized tonic-clonic seizure (GTC). After 6 months, another EEG (with the same previously mentioned protocol) was performed for assessment of the persistence of any epileptiform discharge. 


\section{Classification of patients}

After the follow-up period, patients were classified into three groups: group I $(n=10)$ included patients who did not develop any major or minor seizure during the follow-up period and their follow up EEG was free of any epileptiform discharge, group II $(n=11)$ included patients who developed only minor seizures and their follow up EEG showed epileptiform discharge, and group III $(n=9)$ who developed one or more major seizures during the follow-up period whatever their EEG findings.

Periodic limb movement index and frequency were compared among the three groups of patients and the control group. Periodic limb movement indices were also evaluated in correlation with different clinical characteristics of patients.

\section{Statistics}

Data were fed to the computer and analyzed using IBM SPSS software package version 20.0 [15]. Qualitative data were described using number and percent. Quantitative data were described using range (minimum and maximum), mean, standard deviation, and median. The significance of the obtained results was judged at the 5\% level [16]. The used tests were the chi-square test that was used for comparing categorical variables between different groups, Student's $t$ test used for comparing normally quantitative variables between two studied groups, and $F$ test (ANOVA) for comparing normally quantitative variables between more than two groups. Mann Whitney test was used for comparing abnormally quantitative variables between two studied groups. Kruskal-Wallis test was used to compare abnormally quantitative variables between more than two studied groups. Spearman coefficient was used to correlate between two abnormally quantitative variables.

\section{Results}

\section{Demographic data}

This study was carried out on 30 patients: 19 female (63\%) and 11 males (37\%). The median age was 14.5 years (range from 9 to 29 years), and the mean age at onset of epilepsy was $13.53 \pm 3.79$ (range from 8 to 21 years). Thirty healthy individuals were recruited as control cases: 14 females (53.3\%) and 16 males (46.7\%). The median age was 17 years with a range from 9 to 28 years. Both patients and control were matched for gender and age, and the difference between them was not statistically significant. The mean body mass index (BMI) of the studied groups was $(22.60 \pm 5.03),(21.98 \pm 3.85)$, $(22.02 \pm 3.91)$, and $(23.29 \pm 3.29)$ for group I, group II, group III, and the control group; respectively. The difference between the groups was not statistically significant $(p=0.937)$.

\section{Clinical characteristics of the patients}

Among the 30 studied patients, 19 were diagnosed with juvenile myoclonic epilepsy (JME) (63.3\%), 7 were diagnosed with epilepsy with generalized tonic-clonic seizures (GTCs) only (23.3\%), 2 were diagnosed with juvenile absence epilepsy (JAE) (6.7\%) and 2 were diagnosed with childhood absence epilepsy (CAE) (6.7\%). Family history of epilepsy was positive in $17 \%$ patients (56.7\%). The first EEG was positive in 23 patients (76.7\%).

The mean age at onset of epilepsy for all studied patients was $13.53 \pm 3.79$ years, and for the three subgroups, the age at onset was $15.90 \pm 3.75$ years among group I, $12.82 \pm 3.28$ years among group II, and $11.89 \pm 3.37$ years among group III. The difference between the three subgroups was statistically significant $(p=0.042)$, with group III having the youngest age at onset, followed by the group II; while group I had the eldest age at onset.

The frequency of seizures before treatment was assessed in this study through history taking and from patient's diaries. The frequency was measured per months and compared among the studied patients. Among the three patient groups, the median frequency of seizures at onset (before treatment) was $0.85,0.21$, and 0.35 seizure per month among group I, II, and III, respectively. The frequency of major seizures before treatment was highest among group I and lowest among group II. However, the difference was not statistically significant $(p=0.115)$.

The sleep quality was assessed using PSQI questionnaire. Individuals who scored 5 or more were considered to have a poor sleep quality. Thirty percent of group I, $18.2 \%$ of group II, $22.2 \%$ of group III, and $10 \%$ of the control group had poor sleep. The mean PSQI values were 4.6, 4.18, 3.89, and 3.37 for each of the four groups, respectively. In spite of the different values, none of them were of statistical significance.

\section{Sleep measures}

All recruited subjects and controls completed an overnight PSG. Sleep data are presented in Table 1. The mean total sleep time was not significantly different between the patients' groups and controls [341 min for group I (range 219-447 min), $339 \mathrm{~min}$ (range 309-492 min) for group II, $412 \mathrm{~min}$ for group III (range 272-478), and $391 \mathrm{~min}$ for controls (range 284-552) $(p=0.311)]$. Sleep efficiency had comparable values among patients and control with no statistically significant difference. The mean sleep efficiency was $94 \%, 93 \%, 97 \%$, and $96.83 \%$ among group I, group II, group III, and controls, respectively. Stage 1 sleep percentage and REM sleep percentage were statistically different among the groups. Group II had the highest stage 1 sleep percentage in comparison to the other two groups and the control $(p=0.003)$. No significant differences were found in stage II sleep percentage, slow-wave sleep percentage, wakefulness after sleep onset (WASO), 
Table 1 Comparing demographic and sleep parameters among patients and controls

\begin{tabular}{|c|c|c|c|c|c|}
\hline & $\begin{array}{l}\text { Group I } \\
(n=10)\end{array}$ & $\begin{array}{l}\text { Group II } \\
(n=11)\end{array}$ & $\begin{array}{l}\text { Group III } \\
(n=9)\end{array}$ & $\begin{array}{l}\text { Control } \\
(n=30)\end{array}$ & $p$ value \\
\hline Male (\%) & $30 \%$ & $45.5 \%$ & $33.3 \%$ & 46.7 & 0.741 \\
\hline Age (in years) & $17.5(9-24)$ & $13(11-29)$ & $14(10-18)$ & $17(9-28)$ & 0.356 \\
\hline $\mathrm{BMI}\left(\mathrm{kg} / \mathrm{m}^{2}\right)$ & $21(17.8-35.5)$ & $22.1(18-31.2)$ & $23.4(17.2-27)$ & $23.3(18-32.7)$ & 0.937 \\
\hline Age at onset of epilepsy (years) & $15.90(9-22)$ & $12.82(10-20)$ & $11.89(8-17)$ & - & $0.042^{*}$ \\
\hline Frequency of seizures at onset of presentation per month & $0.85(0.03-8)$ & $0.21(0.11-1.4)$ & $0.35(0.11-2.1)$ & - & 0.115 \\
\hline Positive family history (\%) & 60 & 45.5 & 66.7 & - & 0.660 \\
\hline PSQI & $4.6(1-12)$ & $4.18(1-11)$ & $3.89(1-12)$ & $3.37(1-13)$ & 0.880 \\
\hline PSQI > $5(\%)$ & 30 & 18.2 & 22.2 & 10 & 0.874 \\
\hline Positive first EEG (\%) & 70 & 72.7 & 88.9 & - & 0.650 \\
\hline Total sleep time (min) & 342 (219-447) & 339 (309-492) & 412 (272-478) & $391(284-552)$ & 0.311 \\
\hline Sleep latency (min) & $3.5(0.5-31)$ & $8(10-16)$ & $5(0.5-8)$ & $5.73(1.0-11.5)$ & 0.0 .368 \\
\hline Sleep efficiency (\%) & $94(80-98)$ & $93(89-100)$ & 97 (91-98) & $96.83(91-100)$ & 0.230 \\
\hline REM latency (min) & $19.25(5-244)$ & $118(7-213)$ & $118(12-211)$ & $85(3.50-102.0)$ & 0.063 \\
\hline Stage 1 sleep (\%) & $2.0(1.0-9.0)$ & $8.0(1.0-12.0)$ & $1.0(1.0-5.0)$ & $2.0(1.0-4.0)$ & $0.003^{*}$ \\
\hline Stage 2 sleep (\%) & $52.90(36-71)$ & $46.27(33-73)$ & $53.44(35-59)$ & $54.40(37-70)$ & 0.420 \\
\hline Slow-wave sleep (\%) & $27(12-43)$ & $37(11-44)$ & $35(21-62)$ & $27.0(1.0-47)$ & 0.192 \\
\hline REM (\%) & $21(13-25)$ & $9(5-29)$ & $8(3-15)$ & $18(10-28)$ & $0.015^{*}$ \\
\hline Arousal index/h & $14(4.7-28)$ & $16.8(11.4-24)$ & $13(6-28)$ & $9.6(6.0-31.5)$ & 0.460 \\
\hline PLM index & $0.85(0.1-17.8)$ & $10.8(1.5-23.8)$ & $15.1(0.8-23.8)$ & $2.8(0.2-45.7)$ & $0.010^{*}$ \\
\hline PLM index $>5(\%)$ & 20 & 45.5 & 77.6 & 6.7 & $0.046^{*}$ \\
\hline
\end{tabular}

$B M I$ body mass index, $P p$ value for comparing between the studied groups, $P L M$ periodic limb movement, PSQ/ Pittsburgh Sleep Quality Index, REM rapid eye movement, *Statistically significant at $p \leq 0.05$

total arousal index, and REM arousal index among patient groups and controls. REM sleep percentage was significantly shorter among groups II and III, with median values of 9 and 8 , respectively $(p=0.015)$. The median REM duration in group I was $21 \%$ which was comparable to the control value of $18 \%$. The REM latency was 118 min in both groups II and III which was longer than group I $(19.25 \mathrm{~min})$ and the controls $(85 \mathrm{~min})$. However, the difference was not statistically significant $(p=0.063)$.

As regards periodic limb movement (PLM), the number of individuals who had periodic limb movement index more than 5 was significantly higher in patients $(46.7 \%)$ than in controls $(10 \%)(p<0.001)$. Among the studied subgroups, the difference was statistically significant as regards both the periodic limb movement indices and the number of patients with indices more than 5 among each group. Twenty percent of group I, $45.5 \%$ of group II, $77.6 \%$ of group III, and $6.7 \%$ of the control group had PLM $>5$. The difference between the groups was statistically significant $(p=0.046)$ (Fig. 1$)$. The median periodic limb movement index was $0.85,10.86$, 15.1, and 2.8 among groups I, II, III, and the control group; respectively. This difference was also statistically significant $(p=0.010)$. Group III had the highest PLM index and the highest frequency of PLM indices $>5$.

\section{Correlations}

PLM was evaluated in correlation with different clinical characteristics of patients including age at onset of epilepsy, types of seizures at onset of seizures (minor or major seizure), frequency of seizures before treatment, and family history of epilepsy, significant correlation was observed between the PLM and age at onset of epilepsy. Periodic limb movement also was inversely correlated with the age of onset at epilepsy. The more the periodic limb movement index, the younger the patient developed seizures (Fig. 2).

\section{Discussion}

Periodic limb movements were more prevalent among patients with genetic generalized epilepsy than controls in this study. In agreement with this result, Malow et al. [2] reported increased frequency of periodic limb movement among epileptic patients. Similarly, Khatami et al. [17], El-Fatatry et al. [18] and El-Bahrawy et al. [19] reported increased periodic limb movement index among patients with idiopathic generalized epilepsy. Additionally, this study showed that there was a significant correlation between periodic limb movements and seizure recurrence on follow-up. It was noted that patients with recurrent seizures and poor response to treatment had frequent 


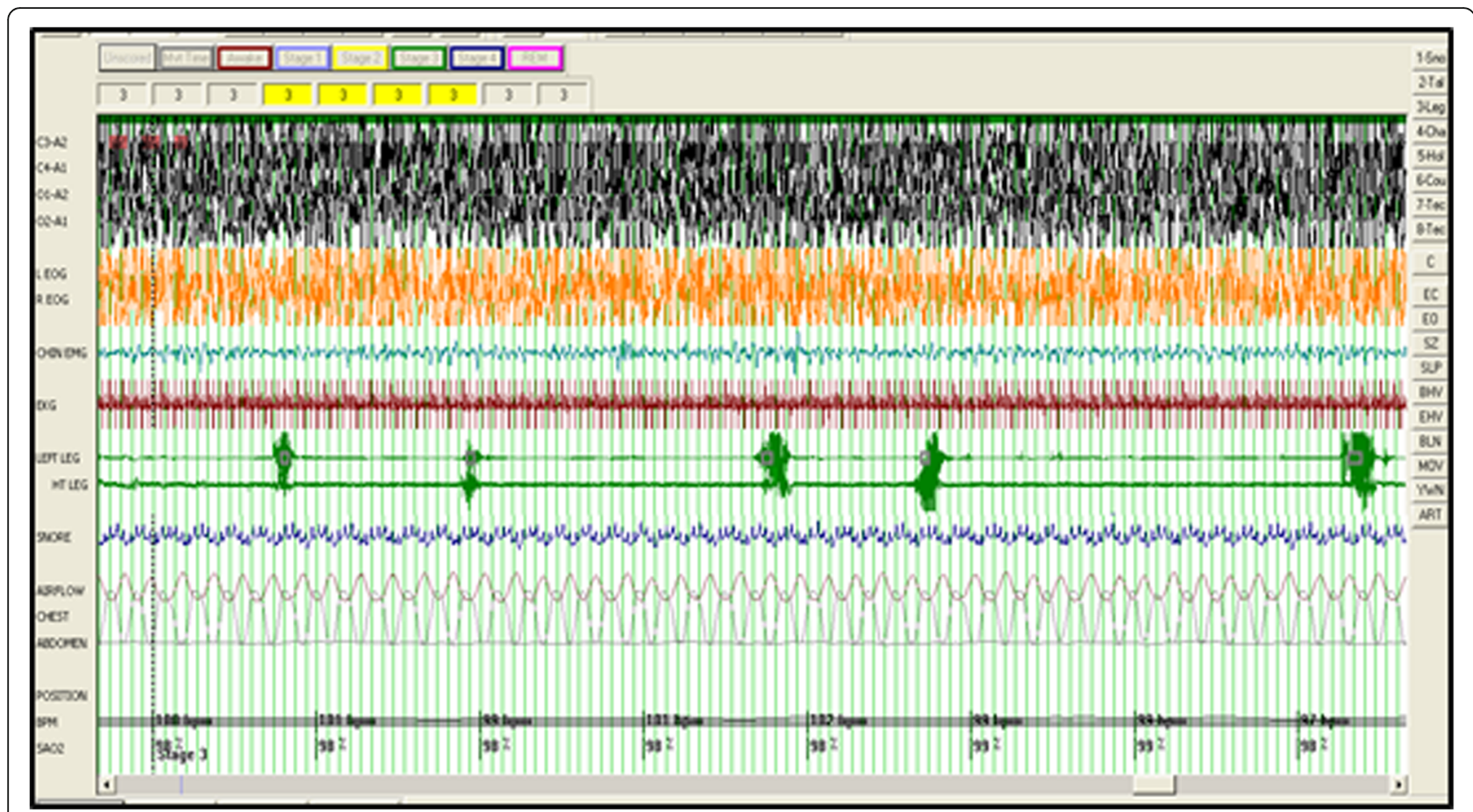

Fig. 1 Overnight polysomnography showing periodic limb movement during NREM sleep in an 11-year old girl with CAE

PLM. The PLMs also seemed to be correlated with the age at onset of epilepsy. However, it cannot be concluded whether the PLMs is a direct cause of early presentation of epilepsy and experiencing recurrent seizures or their occurrence together is just an epiphenomenon.
Researches demonstrated that periodic limb movements were associated with EEG and autonomic oscillations occurring during the cyclic alternating pattern which occurs mainly during NREM sleep [20]. These alternations are referred to as "cyclic alternating pattern";

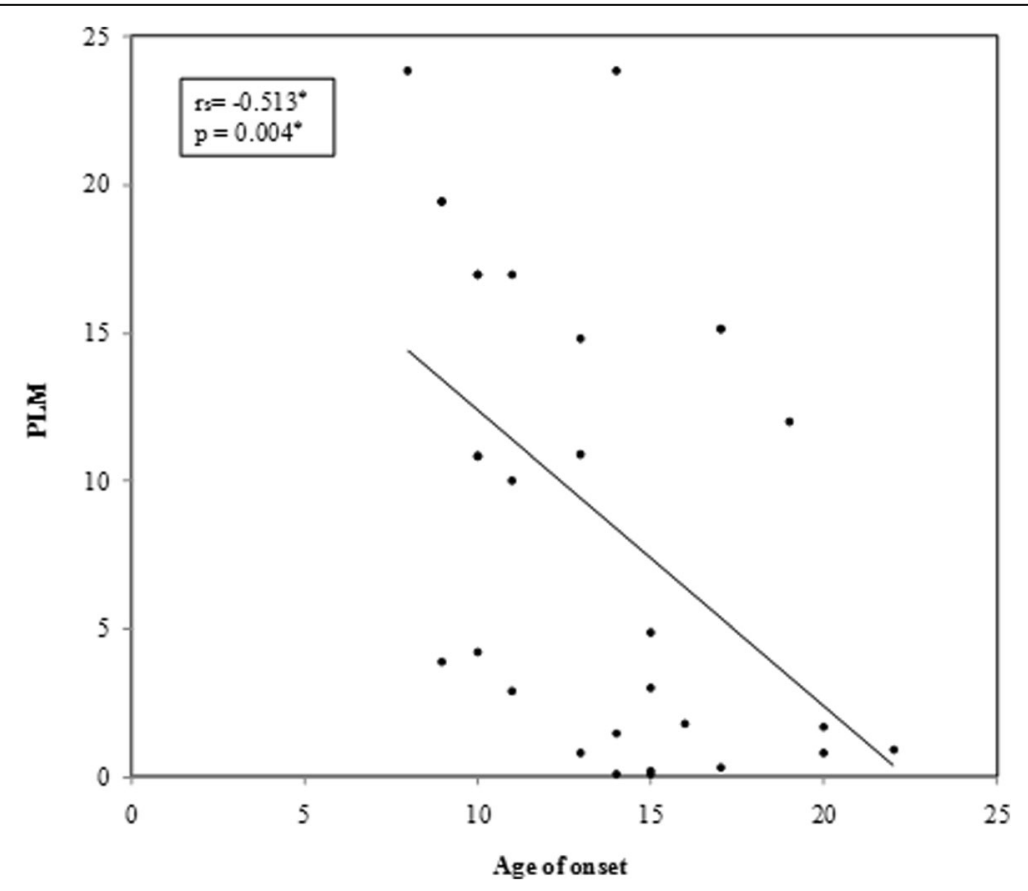

Fig. 2 Correlation between age at onset of epilepsy and periodic limb movement index 
each cycle is composed of two phases: phase A and phase B. Phase B represents the background rhythm, while phase A refers to the phasic events. This cyclic alternating pattern (CAP) is known to be a direct facilitator to interictal epileptiform discharge and clinical seizures during phase A [21]. So, it could be the frequency of CAP during sleep that resulted in the occurrence of both the periodic limb movements and the clinical seizure. Further studies are recommended to study the role of CAP in predicting the seizure recurrence and outcome of epilepsy. However, the periodic limb movement index can still be a good early predictor of seizure recurrence indirectly.

\begin{abstract}
Abbreviations
AED: Anti-epileptic drugs; ALT: Alanine aminotransferase; AST: Aspartate aminotransferase; BMI: Body mass index; CAE: Childhood absence epilepsy; CBC: Complete blood count; CT: Computer tomography;

EEG: Electroencephalography; EMG: Electromyography; GGE: Genetic generalized epilepsy; GTCs: Generalized tonic-clonic seizures; IED: Interictal epileptiform discharge; JME: Juvenile myoclonic epilepsy; NREM: Non-rapid eye movement; PLM: Periodic limb movements; PSG: Polysomnography; PSQI: Pittsburgh Sleep Quality Index; REM: Rapid eye movement; TIB: Time in bed

\section{Funding}

None.

\section{Availability of data and materials}

The dataset can be available with authors' approval.

\section{Authors' contributions}

$\mathrm{MMH}$ participated in creating the idea and principle of the conducted research and he recruited the cases for research. AME created the study design, methodology, and revised the writing process. JFM was the sleep scorer who scored the polysomnographic data for all patients, supervised and revised the written material. EH followed up the recruited patients, wrote the paper material, conducted the follow-up EEG, and she is the corresponding author for editing. All authors read and approved the final manuscript.
\end{abstract}

\section{Ethics approval and consent to participate}

Written informed consent from the patient, or in the case of children, their parent or legal guardian was given to publish the data, and the research was approved by the Ethics Committee of the Faculty of Medicine - Alexandria University (which is constituted and operates according to ICH GCP guidelines and applicable local and institutional regulations and guidelines which govern EC operation for the last 9 years. It has FWA from 2010). The ethical approval was taken on May 2015, and the reference numbers are [1KB NO: 00007555 and FWA NO: 00015712].

\section{Consent for publication}

All participants had signed an informed consent to participate and for the data to be published.

\section{Competing interests}

The authors declare that they have no competing interests.

\section{Publisher's Note}

Springer Nature remains neutral with regard to jurisdictional claims in published maps and institutional affiliations.

Received: 25 November 2017 Accepted: 25 March 2019

Published online: 29 May 2019

\section{References}

1. Bazil CW. Sleep and epilepsy. Semin Neurol. 2017;37(4):407-12.
2. Malow BA, Fromes GA, Aldrich MS. Usefulness of polysomnography in epilepsy patients. Neurology. 1997;48(5):1389-94 Available from: http:// www.ncbi.nlm.nih.gov/pubmed/9153478 [cited 15 Sep 2016].

3. Yazdi Z, Sadeghniiat-Haghighi K, Naimian S, Zohal MA, Ghaniri M. Prevalence of sleep disorders and their effects on sleep quality in epileptic patients. Basic Clin Neurosci. 2013:4(1):36-41.

4. Vetrugno R, D'Angelo R, Montagna P. Periodic limb movements in sleep and periodic limb movement disorder. Neurol Sci. 2007;28(SUPL.1):S9-14.

5. Karadeniz O, Besset B. EEG arousals and awakenings in relation with periodic leg movements during sleep. J Sleep Res. 2000;9(3):273-7.

6. Parrino L, Ferri R, Bruni O, Terzano MG. Cyclic alternating pattern (CAP): the marker of sleep instability. Sleep Med Rev. 2012;16(1):27-45.

7. Allena M, Campus C, Morrone E, De Carli F, Garbarino S, Manfredi C, et al. Periodic limb movements both in non-REM and REM sleep: relationships between cerebral and autonomic activities. Clin Neurophysiol. 2009;120(7): 1282-90.

8. Kohrman MH. Epilepsy and sleep. J Pediatr Epilepsy. 2013;2(3):183-97.

9. Berg AT, Millichap JJ. The 2010 revised classification of seizures and epilepsy. Continuum Lifelong Learn Neurol. 2013;19:571-97.

10. Fisher RS, Acevedo C, Arzimanoglou A, Bogacz A, Cross JH, Elger CE, et al. ILAE official report: a practical clinical definition of epilepsy. Epilepsia. 2014; 55(4):475-82.

11. Jagannath A, Peirson SN, Foster RG. Sleep and circadian rhythm disruption in neuropsychiatric illness. Curr Opin Neurobiol. 2013;23:888-94.

12. Flink R, Pedersen B, Guekht a B, Malmgren K, Michelucci R, Neville B, et al. Guidelines for the use of EEG methodology in the diagnosis of epilepsy. International league against epilepsy: commission report. Commission on European affairs: subcommission on European guidelines. Acta Neurol Scand. 2002;106:1-7.

13. American Academy of Sleep Medicine. International classification of sleep disorders: diagnostic and coding manual, vol. 281, Diagnostic Coding Manual. 3nd ed; 2014. p. 2313.

14. Glauser T, Ben-Menachem E, Bourgeois B, Cnaan A, Guerreiro C, Kälviäinen $R$, et al. Updated ILAE evidence review of antiepileptic drug efficacy and effectiveness as initial monotherapy for epileptic seizures and syndromes. Epilepsia. 2013;54(3):551-63.

15. Kirkpatrick LA. A simple guide to IBM SPSS statistics for version 20.0. Student ed. Belmont: Wadsworth, Cengage Learning; 2013.

16. Kotz S, Balakrishnan N, Read CBVB. Encyclopedia of statistical sciences. 2nd ed. Hoboken: Wiley-Interscience; 2006.

17. Khatami R, Zutter D, Siegel A, Mathis J, Donati F, Bassetti CL. Sleep-wake habits and disorders in a series of 100 adult epilepsy patients-a prospective study. Seizure. 2006;15(5):299-306.

18. Al-Fatatry. A comprehensive study of interrelation between sleep and epilepsy. MD [dissertation]. Alexandria: Alexandria University; 2009.

19. ElBahrawy S, Mekky J, Saadallah HBM. Sleep study in patients with juvenile myoclonic epilepsy: newly diagnosed and those on valproate. MS [dissertation]. Alexandria: Alexandria University; 2016.

20. Parrino L, Boselli M, Buccino GP, Spaggiari MC, Di Giovanni G, Terzano MG. The cyclic alternating pattern plays a gate-control on periodic limb movements during non-rapid eye movement sleep. J Clin Neurophysiol. 1996;13(4):314-23.

21. Parrino L, Smerieri A, Spaggiari MC, Terzano MG. Cyclic alternating pattern (CAP) and epilepsy during sleep: how a physiological rhythm modulates a pathological event. Clin Neurophysiol. 2000;111(SUPPL. 2):S39-46. 